\title{
USE OF DEEP OSCILLATION - THERAPY IN REHABILITATION PROGRAM FOR PATIENT AFTER DISTAL RADIUS FRACTURE WITH A COMPLEX REGIONAL PAIN SYNDROME: A CASE REPORT
}

\author{
G. Mratskova* \\ Department of Medical Rehabilitation and Ergotherapy, Physical Medicine and Sports, \\ Medical Faculty, Trakia University, Stara Zagora, Bulgaria
}

\begin{abstract}
THE PURPOSE is to present a clinical case of a patient with a fracture of the distal radius and complex regional pain syndrome I, in which complex rehabilitation was applied, including Deep Oscillation, cryotherapy and therapeutic exercises.

MATERIALS AND METHODS: The rehabilitation program was conducted to a 62-year-old woman, four months after the injury. The intervention includes Deep Oscillation in biphasic mode (up to $7 \mu \mathrm{A}$ ) and therapeutic exercises 10 procedures. The clinical symptoms were assessed before and after therapy and after 1-st and 3-rd months after therapy.

RESULTS: Reduction in pain (VAS) after therapy from "very strong" (75mm) before therapy, to "mild" at 3 months (12mm) was observed. Reduction of wrist swelling and muscle weakness as assessed by manual muscle testing was observed. The range of movement in the sagittal plane from $25^{\circ}-0-35^{\circ}$ to $70^{\circ}$ $0^{\circ}-85^{\circ}$ and the frontal plane from $5^{\circ}-0-15^{\circ}$ to $20^{\circ}-0^{\circ}-35^{\circ}$ was increase. Improved performance of activities of daily living was observed. No adverse events with Deep Oscillation therapy were observed.

CONCLUSION: Deep Oscillation and therapeutic exercises effectively reduce the clinical symptoms of regional pain syndrome. To establish the therapeutic efficacy of Deep Oscillation treatment after a fracture of the distal radius with complex regional pain syndrome, randomized studies involving a larger number of patients are required.
\end{abstract}

Key words: Low frequency and low intensity pulsation electrostatic field, Sudeck atrophy, kinesitherapy, cryotherapy, activities of daily living.

\section{INTRODUCTION}

Distal radius fractures (FDRs) are common in patients over 60 years of age with frequency second most common after hip fractures $(1,2)$. According to some authors, they represent $15 \%$ to $18 \%$ of all fractures $(3,4)$. In adult the most common causes are low-energy trauma (5), while in young patients the most common cause is high-energy trauma (6).

Although the incidence varies worldwide, FDRs are common throughout life and are likely to continue to increase. The risk is

\footnotetext{
*Correspondence to: Galina Mratskova, Department of Medical Rehabilitation and Ergotherapy, Physical Medicine and Sports, Medical Faculty, Trakia University, Stara Zagora, Bulgaria, Str. ,,Armeiska“ № 11 GSM +359894 781 999, e- mail: galina.mratskova@trakia-uni.bg
}

determined by a number of personality factors (age, gender, lifestyle, health) and environmental factors. Worse health outcomes are associated with old age, female gender, poor bone healing, etc. (5). The increase in prevalence is probably associated with an increase in life expectancy (7). In menopausal and postmenopausal women, even a minor injury can cause FDR. The frequency increases with age, with a peak with between 60 and 65 years of age (8).

Complex regional pain syndrome (CRPS) is a common complication after FDRs, which occurs in the first four months after the trauma. It is more common in women than in men, with a ratio of 3, 5:1 (9). Its frequency varies from $22 \%$ to $39 \%$. CRPS can complicate recovery and reduce health-related quality of life (10). 
The involvement of central and peripheral mechanisms, changes in sympathetic afferent transmission, inflammatory and immune changes, genetic and psychological factors are probably responsible for the occurrence of CRPS. The syndrome is often diagnosed based on clinical signs and symptoms (11). The central sensitization is considered to be the main reason for the development of CRPS (12).

Table 1. Clinical diagnostic criteria for CRPS (International Association for the Study of Pain (IASP)) (17):

\begin{tabular}{|c|c|}
\hline 1. & Continuing pain, which is disproportionate to any inciting event. \\
\hline 2. & $\begin{aligned} & \text { Must report at least one symptom in three of the four of the following categories: } \\
& \checkmark \text { Sensory: reports of hyperaesthesia and/or allodynia } \\
& \checkmark \text { Vasomotor: reports of temperature asymmetry and/or skin colour changes and/or } \\
& \text { skin colour asymmetry } \\
& \checkmark \text { Sudomotor/oedema: reports of oedema and/or sweating changes and/or sweating } \\
& \text { asymmetry } \\
& \checkmark \text { Motor/trophic: reports of decreased range of motion and/or motor dysfunction } \\
& \checkmark \text { (weakness, tremor, dystonia) and/or trophic } \\
& \text { changes (hair, nail, skin) }\end{aligned}$ \\
\hline 3. & $\begin{array}{l}\text { Must display at least one sign at time of evaluation in two or more of the following } \\
\text { categories: } \\
\checkmark \text { Sensory: evidence of hyperalgesia (to pinprick) and or allodynia (to light touch } \\
\text { and/or deep somatic pressure and/or joint } \\
\checkmark \text { movement) } \\
\checkmark \text { Vasomotor: evidence of temperature asymmetry and/or skin colour changes and/or } \\
\text { asymmetry } ~ \text { Sudomotor/oedema: evidence of oedema and/or sweating changes } \\
\text { and/or sweating asymmetry - Motor/trophic: evidence of a decreased range of } \\
\text { motion and/or motor dysfunction (weakness, tremor, dystonia) and/or trophic } \\
\checkmark \text { changes (hair, nail skin) }\end{array}$ \\
\hline
\end{tabular}

4. There is no other diagnosis that better explains the signs and symptoms.

CRPS I (reflex sympathetic dystrophy or algodystrophy) is a chronic neuropathic condition characterized by regional spontaneous or induced pain, tactile hypersensitivity, edema, vasomotor changes and impaired motor function $(16,17)$. Three stages of flow are described. The first stage is acute inflammation. It starts about 10 days after the injury and lasts 3-6 months. It is characterized by sympathetic dysfunction and dermatome-type pain propagation. The pain is more severe than expected. There is a local soft edema with hyperemia and thermal changes of the skin - warming, increased growth of hair and nails. In the second stage there is a progression of dystrophy, hard edema, vasoconstriction, hyperhidrosis, neurovascular instability, brittle nails, and changes in temperature and skin color. Radiologically, osteoporosis and subchondral bone erosion were found. In the third stage,
Two different types of CRPS have been described in the literature: CRPS I - occurs without evidence of nerve damage and CRPS II - (formerly called causalgia), occurs with proven prior nerve damage (13-16). Clinical diagnostic criteria for CRPS (International Association for the Study of Pain (IASP)) (15) are presented in Table 1. 
TENS (14) and Medium-frequency interference currents may also be used. Local application of low frequency currents and irritating procedures is not recommended in CRPS.

Deep Oscillation therapy based on low frequency (5 to $250 \mathrm{~Hz}$ ) and low intensity pulsation electrostatic field, an electromagnetic field with low, non-thermal intensity $(21,22)$. The physiological mechanism of action consists in intense resonant vibration of the tissues in depth, which are located below the applicator when moving in the sagittal direction (23). The physiological effects are frequency dependent. The intensity of the current is small 5-7 $\mu \mathrm{A}$. It is applied in the form of biphasic pulses. The effects of electrolysis are not obtained. During each pause, the field is diluted and the possibility of accumulation of static electricity in the patient's body is excluded (22).

\section{PURPOSE OF THE STUDY}

The aim of this article is to present a clinical case of a patient with a fracture of the distal radius and complex regional pain syndrome I, without peripheral nerve damage, in which complex rehabilitation program was applied, including Deep Oscillation, cryotherapy and therapeutic exercises.

\section{MATERIALS AND METHODS}

The clinical case described in this article is of a 62-year-old woman who falls from a standing position and has a fracture of the distal radius of her right wrist.

Non-operative repositioning and plaster cast immobilization were performed. No neurological deficit was determined. After 24 hours, due to extraordinarily strong edema and pain, the plaster bandage was replaced with a new one. On the 6th day, a new reposition and immobilization were performed due to crepitation under the plaster cast immobilization. The plaster immobilization was removed after 30 days. The patient is not referred for rehabilitation. Guidelines for physical activity at home were given. After 4 months, due to severe pain, swelling and limited movement, the patient was consulted by an orthopedist and an X-ray with evidence of osteoporosis was performed. CRPS I have been diagnosed. Although according to the IASP criteria, the change in bone density is not a diagnostic criterion; its significance has been reported in previous studies as being significant for CRPS. She is referred to a specialist in Physical and Rehabilitation Medicine (PRM) for rehabilitation.
At the first examination by a PRM specialist, the patient reported severe pain, extreme sensitivity to touch, swelling of the arm, inability to perform active movements. The physical examination revealed data for local trophic changes: changes in the skin color, a slight warming of the hand, edema of the wrist joint, dorsum manus dextra and fingers with moderate density. Presence of spontaneous and palpable pain at rest, severe pain during active movements and limited range of active movements for articulatio: radiocarpalis, radioulnaris, cubiti, metacarpophalangealis, intrerphalangealis. There is extremely limited use of the hand in daily activities.

Methods for assessment: To determine the rehabilitation potential, a Visual Analog Scale VAS ( $\mathrm{mm}$ ) is used to assess pain, measuring the circumference of the wrist and fingers by centimetry, range of motion test (SFTR), manual muscle testing (MMT). Clinical symptoms were assessed before and after therapy after 1 and 3 months.

A Visual Analog Scale (VAS), measured in $\mathrm{mm}$, is used to assess pain. It is a onedimensional scale that is widely used in different age populations. VAS is a straight line from 0 to $10 \mathrm{~cm}(0 \mathrm{~mm}$ to $100 \mathrm{~mm})$, at the beginning and in the end, on which there are two descriptors representing extreme values of pain intensity (0- no pain and 10 extremely severe pain) at each end, 2 - mild pain, 4moderate pain, 6-severe pain, 8-very severe pain. Patients themselves determine their pain intensity by placing a mark on the line that represents their pain intensity.

\section{Measurement of circumferences -}

Santimetry is a kinesiological method for verification of anthropometric indicators. A plastic centimeter is used to determine the development of the muscles in normal and pathology /muscle atrophy/, joint circumference - normal, pathology /swelling /.

Manual Muscle Testing (MMT) A test method for determining the degree of muscle weakness due to disease, injury or hypokinesia. MMT assessments allow to build an adequate kinesitherapy program for diseases of the musculoskeletal system. Muscle weakness is assessed in six grades with grades from 0 to 5 . Grades in MMT 5, 4 and 3 are called functional. In some cases, the test results may not exactly match the definitions given for the six grades. Adjustment is allowed by adding a + or - sign to 
the respective assessment. It is assumed that each of these signs corresponds to $5-10 \%$ strength. (3+) - movement against gravity performed several times or once against slight resistance; (3-) - movement against gravity performed in an incomplete range of motion of $50-90 \%$ of the range of the movement; $(2+)$ - starting movement against gravity - $50 \%$ of the range of the motion or less; (2-) - movement in incomplete range with eliminated gravity.

In order to objectify the results from MMT test application in cases where the signs "+" or "-" are used to the corresponding number used to quantify muscle weakness, presented in Table 2.

Table 2. Degrees of muscle weakness according to the manual muscle test (MMT)

\begin{tabular}{|c|c|c|c|c|c|c|c|c|c|c|}
\hline MMT & 2 & $2+$ & 3- & 3 & $3+$ & 4- & 4 & $4+$ & $5-$ & 5 \\
\hline & & 2.25 & 2.75 & 3 & 3.25 & 3.75 & 4 & 4.25 & 4.75 & 5 \\
\hline
\end{tabular}

Range of motion. It is the main parameter for determining motor function, the test is performed by goniometry in angular degrees to determine the range of motion (SFTR) the joints.

Based on the conducted physical examination and specific tests, an individual rehabilitation program was prepared. The main goal of the rehabilitation program is: Optimal functional recovery of the right arm. Objectives of the rehabilitation program are: Reducing the pain syndrome, edema and the local inflammatory response, increasing the range of motion in the affected joints, reducing muscle weakness, prevention of contractures and inclusion of upper limb in activities of daily living.

Intervention: The treatment was applied as a course - 10 days, 10 procedures of the type, of course in ambulatory physical and rehabilitation department (Monday to Friday): Deep Oscillation therapy, ice block cryotherapy, and a session of therapeutic exercises. The rehabilitation program was carried out in accordance with the instructions of the Declaration of Helsinki (1964) and with the written consent of the patient.

Application technique: Deep Oscillation therapy is applied by means of a manual applicator (oscillating head) $-5.5 \mathrm{~cm}$. The procedure includes treatment of the wrist joint, surrounding tissues, fingers, antebrachium and brachium. Therapeutic modalities: Variable frequency $100-200 \mathrm{~Hz} 15 \mathrm{~min}$. Modulation mode 1: 1 (light vibrations).

Cryotherapy with an ice block (frozen at $-20^{\circ}$ ) 5-10 minutes in the wrist area and the fingers, before the session with therapeutic exercises. The hand was dried with a cotton cloth after the intervention.

Therapeutic exercises: Active isotonic, unforced exercises for all joints of the upper limb, up to the limit of pain. Absolute contraindications are passive and pain-provoking movements. Emphasis is placed on the muscles that counteract contractile tendencies (flexors and extensors of the fingers $\mathrm{mm}$. intereossei, extensors and flexors of the wrist, m. delatoideus, $\mathrm{mm}$. supraspinatus, infraspinatus, subscapularis, $\mathrm{m}$ flexsor digitorum, $\mathrm{m}$ extensor digitorum) exercises with a small rubber ball until pain occurs, isotonic and isometric, including resistance exercises for the contralateral upper limb.

\section{RESULTS}

Therapeutic results were monitored in dynamics before the beginning of the rehabilitation course, immediately after it, and 1 and 3 months after treatment. Clinical reduction of the pain syndrome, reduction of edema and improvement of the range of motion were established immediately after the rehabilitation, which was maintained until the end of the 3rd month. The results are presented in Table 3 .

Table 3. Dynamics in the level of pain, swelling and range of motion in the wrist joint page 8

\begin{tabular}{lllll}
\hline Indicator & Before therapy & After therapy & 1 -st month & 3-rd month \\
\hline Pain VAS (mm) & $75 \mathrm{~mm}$ & $38 \mathrm{~mm}$ & $25 \mathrm{~mm}$ & $12 \mathrm{~mm}$ \\
\hline Santimetry & $22,5 \mathrm{~cm}$ & $20,8 \mathrm{~cm}$ & $20,2 \mathrm{~cm}$ & $19,6 \mathrm{~cm}$ \\
\hline Goniometry & & & & \\
\hline Pronation-supination & $25^{\circ}-0^{\circ}-20^{\circ}$ & $55^{\circ}-0^{\circ}-45^{\circ}$ & $65^{\circ}-0^{\circ}-70^{\circ}$ & $70^{\circ}-0^{\circ}-75^{\circ}$ \\
\hline Wrist joint & & & & \\
\hline Extension - flexion & $25^{\circ}-0^{\circ}-35^{\circ}$ & $40^{\circ}-0^{\circ}-55^{\circ}$ & $55^{\circ}-0^{\circ}-75^{\circ}$ & $70^{\circ}-0^{\circ}-85^{\circ}$ \\
\hline Radial-ulnar drainage & $5^{\circ}-0^{\circ}-15^{\circ}$ & $10^{\circ}-0^{\circ}-25^{\circ}$ & $20^{\circ}-0^{\circ}-30^{\circ}$ & $20^{\circ}-0^{\circ}-35^{\circ}$ \\
\hline
\end{tabular}


Reduction of muscle weakness was observed according to MMT for the motor muscles of the wrist and fingers (Table 4).

Table 4. Dynamics of manual muscle testing (MMT)

\begin{tabular}{lllll}
\hline $\begin{array}{l}\text { MMT } \\
\text { dynamics }\end{array}$ & $\begin{array}{l}\text { Flexion with } \\
\text { radial } \\
\text { abduction- } \\
\text { flexsor carpi } \\
\text { radialis }\end{array}$ & $\begin{array}{l}\text { Flexion with } \\
\text { ulnar drainage- } \\
\text { m. flexsor carpi } \\
\text { ulnaris }\end{array}$ & $\begin{array}{l}\text { Extension with } \\
\text { radial drainage- } \\
\text { m. extensor } \\
\text { carpi radialis } \\
\text { longus et brevis }\end{array}$ & $\begin{array}{l}\text { Extension with } \\
\text { ulnar drainage- } \\
\text { m. ulnaris carpi }\end{array}$ \\
\hline Baseline & $3-$ & 3 & $2+$ & 3 \\
\hline After therapy & 3 & $3+$ & $3-$ & 3 \\
\hline 1-st month & $3+$ & $4-$ & $3+$ & $3+$ \\
\hline 3-rd month & 4 & 4 & $4-$ & $4-$ \\
\hline
\end{tabular}

\section{DISCUSSION}

Fractures in the area of the distal radius DRF are common in menopausal women. For various reasons, they are often accompanied by CRPS. It is one of the most disabling conditions in older women and is a serious challenge for rehabilitation (8). For optimal recovery and rehabilitation of DRF, it is necessary to know the epidemiology of the disease, the factors causing injury, the occurrence of chronic pain and subsequent disability (5). If left untreated, these impairments can lead to functional limitations and impairment (14).The individual rehabilitation program needs to consider the specific clinical symptoms and to be correspondent to the specific phase of the disease.

Currently there is no consensus on the efficacy of the physical factors applied. Although according to some randomized controlled studies, physiotherapy and occupational therapy are effective in treating CRPS. There are a number of studies that report a positive effect from the application of low-frequency Diadynamic currents, TENS, Mediumfrequency currents, Magnetic field, Laser therapy and light therapy (8).

Based on the information that the low frequency and low intensity pulsation electrostatic field causes a reduction in pain and swelling, Deep Oscillation therapy was included in the patient's individual rehabilitation program. A review of the available literature revealed information about positive therapeutic effects in sports injuries (24).
No scientific reports have been found on the effectiveness of Deep Oscillation therapy in patients with distal radius fracture and CRPS.

After the application of Deep Oscillation therapy in the present clinical case, a reduction of the pain is observed immediately after the completion of the rehabilitation process, which is established also at the end of the observed three-month period. The therapeutic effects are frequency dependent: $80-250 \mathrm{~Hz}$ analgesic, antispasmodic and anti-edematous action; $25-80 \mathrm{~Hz}$ - enhances metabolic and reparative processes in tissues, improves venous flow and lymph circulation; $5-25 \mathrm{~Hz}-$ stimulates local hemodynamics, improves the functional state of the muscles, correction of impaired tissue trophism. (22). The analgesic effect is rapid and has a long-lasting effect (25). Gate control of the pain impulse is activated (26) and the psychological effect of the procedure is manifested (27). An antiinflammatory effect is observed $(38,39)$.

There is a decrease in the circumference of the wrist joint in centimeters, immediately after the course of treatment and until the end of the three-month observation period. It is likely that the low frequency and low intensity pulsation electrostatic field can effectively affect tissue edema. Some authors report an anti-edema and lymphatic drainage effect $(28,30)$. It reduces inefficient shunt blood flow and subsequently improves microcirculation (31).

After the applied complex rehabilitation in the observed clinical case an increase in the range of motion in the wrist joint was found in the three planes of movement. Jahr, S. et al. reported increased shoulder range of motion and pain reduction after Deep Oscillation monotherapy in patients with lymphedema 
following $\mathrm{Ca}$ mame surgery (30). Deep Oscillation therapy is likely to increase tissue elasticity - reducing and / or preventing fibrosis. Joint mobility is improved. Evgeniya Vladeva et al. reported a reduction in edema, pain symptoms, and restoration of range of motion in patients with knee joint replacement after complex treatment with Deep Oscillation and kinesiotherapy (32).

Trophic and regenerative processes are improved based on activation of local mechanisms of tissue blood flow, increase of local microhemodynamics, increase of blood transport functions, improvement of transcapillary metabolism are observed (22).

This article in the present clinical case has the following limitations: the clinical evaluation is based on the determination of rehabilitation potential by applying conventional tests: joint range of motion test measured by goniometry, muscle weakness measured by MMT, pain measured by VAS $(\mathrm{mm})$, which are largely subjective scales. Although, these assessment methods are routinely used in physiotherapy practice. There is no possibility to establish a causal relation between a rehabilitation program, including Deep Oscillation therapy, and reported results.

\section{FINDINGS AND CONCLUSION:}

- Distal radius fractures (FDRs) are common in patients over 60 years of age often in menopausal and postmenopausal women.

- Complex regional pain syndrome (CRPS) is a common complication after FDRs and leads to functional impairment and reduced quality of life.

- The complex treatment of Deep Oscillation therapy, cryotherapy and therapeutic exercises showed good pain control and a significant improvement in wrist range of motion.

- No adverse reactions from the application of a low frequency alternating electrostatic field were observed during and after therapy.

- The rehabilitation program, including Deep Oscillation and therapeutic exercises, effectively reduces the clinical symptoms in the observed patient with regional pain syndrome (CRPSI).

- To establish the therapeutic efficacy of Deep Oscillation treatment after a distal radius fracture with complex regional pain syndrome, randomized studies involving a larger number of patients are required.

\section{REFERENCES}

1. Court-Brown CM, Caesar B. Epidemiology of adult fractures: a review. Injury, 37(8):691-7, 2006.

2. Raittio, L., Launonen, A., Hevonkorpi, T. et al. Comparison of volar-flexion, ulnardeviation and functional position cast immobilization in the non-operative treatment of distal radius fracture in elderly patients: a pragmatic randomized controlled trial study protocol. BMC Musculoskelet Disord, 18, 401,2017.

3. Nellans, KW, Kowalski, E, Chung, KC. The epidemiology of distal radius fractures. Hand Clin. 28(2):113-125, 2012.

4. Smeraglia, F., Del Buono, A., Maffulli, N. Wrist arthroscopy in the management of articular distal radius fractures, British Medical Bulletin, 119(1):157-165, 2016.

5. MacIntyre, NJ, Dewan N. Epidemiology of distal radius fractures and factors predicting risk and prognosis. J Hand Ther, 29(2):136145, 2016.

6. O'neill, T., Cooper, C., Finn, J., Lunt, M., Purdie, D., Reid, D., Rowe, R., Woolf , A., Wallace W. Incidence of distal forearm fracture in British men and women. Osteoporosis Int., 12(7):555-8, 2001.

7. Nellans, K.W., Kowalski, E., Chung, K.C., The epidemiology of distal radius fractures. Hand Clin, 28:113-125, 2012.

8. Zlatkovic-Svenda, M.I., Leitner, C., Lazovic, B., Petrovic, D.M. Complex Regional Pain Syndrome (Sudeck Atrophy) Prevention Possibility and Accelerated Recovery in Patients with Distal Radius at the Typical Site Fracture Using Polarized, Polychromatic Light Therapy, Photobiomodulation, Photomedicine, and Laser Surgery, 37(4): 233-239, 2019.

9. Goebel, A. Complex regional pain syndrome in adults. Rheumatology,50(10):1739-50, 2011.

10.Li, Z., Smith, B.P., Tuohy, C., Smith, T.L., Koman, A.L. Complex regional pain syndrome after hand surgery. Hand Clin,26:281-289, 2010.

11.Bruehl, S. Complex regional pain syndrome BMJ,351:h2730, 2015.

12.Shipton, E., Complex regional pain syndrome - Mechanisms, diagnosis, and management. Current Anaesthesia and Critical Care, 20(5):209-214, 2009. 
13.Wilson, P.R., Stanton-Hicks, M., Harden, N., et al. Progress in Pain Research and Management; No. 32. CRPS: Current Diagnosis and Therapy. Seattle, WA: IASP Press; 2005.

14.Collins, C.K., Physical therapy management of complex regional pain syndrome I in a 14-year-old patient using strain counterstrain: a case report. J Man Manip Ther., 15(1): 25-41, 2007.

15.Harden, R.N., Bruehl, S., Perez, R.S. et al. Validation of proposed diagnostic criteria (the 'Budapest Criteria') for complex regional pain syndrome. Pain,150:268-74, 2010.

16.Bharwani, K.D, Dirckx, M., Huygen, Frank JPM, FIPP FFPMCAI (hon), Complex regional pain syndrome: diagnosis and treatment, BJA Education, 17(8), 262-268, 2017.

17.Jellad, A., Salah, S., Ben Salah Frih Z. Complex regional pain syndrome type I: incidence and risk factors in patients with fracture of the distal radius. Archives of Physical Medicine and Rehabilitation., 95(3):487-492, 2014.

18.Hooshmand H, Phillips E. Spread of complex regional pain syndrome. Vero Beach, Florida. Neurological Associates Pain Management Center.

19.Cohen, H., McCabe, C., Harris, N., Hall, J., Lewis, J., Blake, D.R. Clinical evidence of parietal cortex dysfunction and correlation with extent of allodynia in CRPS type 1. Eur J Pain, 17:527-538, 2013.

20.de Mos, M. Sturkenboom, M.C. Huygen F.J. Current understandings on complex regional pain syndrome. Pain Pract. 9: 8699, 2009.

21.Zehtindjieva, M.G, Ioshinov, B.R., Andonov, D.R., Ilkov, V.S., Bayraktarova, A., Koleva, I.B. Deep Oscillation - a modern additional physical modality for analgesia in patients with back pain. Praemedicus, 29:85-90, 2013.

22.Kulikov, A.G., Kuzovleva, E.V.,"The application of the Low-frequency electrostatic field in the clinical practice." Russian Journal of Physical Therapy Balneothrrapy and Rehabilitation, 4:4453,2013.

23. Onose, G., Daia-Chendreanu et al. Our experience concerning the use of deep oscillation in the therapy of the use of deep oscillation in the therapy of neurologic patients with associated pain and/or rheumatic - degenerative conditions. - The proceedings from the National Conferrence of Neurosurgery and Neurorehabilitation, 2nd edition", Mamaia, Romania, pp 173176; supl. al "Industria Textile Magazine"cotat ISI, Ed. Certex, 2009.

24.Aliyev, R., Clinical effects of the therapy method deep oscillation in treatment of sports injuries. Sportverletz Sportschaden, 23(1):31-4. 2009.

25.Fistetto, G. et al. Deep Oscillation: therapeutic-rehabilitative experiences with a new electrostatic device. - Minerva Med., 102(4):277-288,2011.

26.Trubulski, R. Using the Hivamat 200 system in the treatment of wounds. Rehabilitacia W. Praktice, 1:28-33, 2008.

27.Koleva, I.B., Ioshinov, B.R. and Yoshinov, R.D., Complex Analgesia (Infiltrations and Deep Oscillation) in Patients with Stump Pain and Phantom Pain after Lower Limb Amputation (Double-blind Randomised Controlled Trial of Efficacy) Journal of Advances in Medicine and Medical Research 22(11): 1-17, 2017.

28. Gasbarro, V. et al. Role of Hivamat ${ }^{\circledR}$ (Deep Oscillation) in the treatment for the lymphedema of the limbs. - Eur J Lymphol, 16:13-18, 2006.

29. Mikhalchik, E. et al. Effects on blood parameters of DEEP OSCILLATION ${ }^{\circledR}$. In: 1st International Conference on Skin and Environment, Moscow - St. Petersburg, 59:2005.

30.Jahr, S. et al. Effect of treatment with lowintensity and extremely low-frequency electrostatic fields (Deep Oscillation) on breast tissue and pain in patients with secondary breast lymphoedema. - Journal of Rehabilitation Medicine,40(8): 645-650, 2008.

31.Korkina, L. et al. Treatment of Gynoid Lipodystrophy (Cellulite) with Deep Oscillation $\mathbb{R}$ : A Pilot Clinical Study. - In: 29th Annual Meeting of The Bioelectromagnetics Society, Kanazawa, Japan. Vol. 2. 2007.

32.Vladeva, E., Mihailova, M., Bacheva, D., Todorov I., Our experience with using Deep Oscillation in early rehabilitation of patients with arthoplasty of the knee joint, Scientific Online Resource System, Varna Medical Forum,7(1):66-70, 2018. 